\title{
Approach to the patient with atypical diabetes
}

\author{
Devin W. Steenkamp MD, Sara M. Alexanian MD, Elliot Sternthal MD
}

Competing interests: None declared.

This article has been peer reviewed.

Correspondence to: Devin W. Steenkamp, devin.steenkamp@bmc.org

CMAJ 2014. DOI:10.1503 /cmaj.130185
$\mathrm{T}$ he overarching term "diabetes mellitus" represents a heterogeneous group of metabolic conditions characterized by hyperglycemia. All of these conditions are underpinned by a combination of various insulin secretory defects and impaired insulin action. According to the American Diabetes Association, ${ }^{1}$ diabetes can be broadly classified into four clinical classes: type 1 diabetes, characterized by immune-mediated destruction of the insulin-secreting $\beta$ cells, which usually leads to absolute insulin deficiency and dependence on exogenous insulin; type 2 diabetes, an undefined polygenic disorder with various degrees of insulin resistance preceding progressive insulin secretory defects; gestational diabetes, diagnosed for the first time during pregnancy; and diabetes due to specific causes other than those noted above. This fourth group remains poorly defined, and atypical forms of diabetes often fall into this "catch-all" category.

Type 2 diabetes accounts for over $90 \%$ of cases seen in primary care, ${ }^{1}$ and type 1 diabetes accounts for the majority of the rest (between $5 \%-10 \%$ of all cases). ${ }^{1}$ However, physicians occasionally encounter individuals with impaired glucose metabolism who are lean, lack markers of insulin resistance or the typical type 2 diabetic dyslipidemic profile, are without hypertension or other typical cardiovascular risk factors, and who are not completely insulin dependent. These patients present a diagnostic challenge.

Diagnosing type 2 diabetes is not usually difficult. Progressive hyperglycemia (usually pre-

\section{KEY POINTS}

- Atypical diabetes is increasingly recognized but is often poorly defined and may masquerade as type 1 or type 2 diabetes.

- Recognition of an atypical diabetes phenotype is important in facilitating timely work-up and often leads to referral for confirmation of the specific diagnosis, prognosis and treatment plans.

- Hepatocyte nuclear factor $1 \alpha$ (HNF1A) monogenic diabetes (also known as maturity-onset diabetes of the young [MODY] type 3 ) is the most common single-gene cause of diabetes.

- Ketosis-prone diabetes should be considered in individuals with phenotypic type 2 diabetes who present with insulin deficiency and ketoacidosis.

- Latent autoimmune diabetes of adulthood constitutes slowly progressive autoimmune diabetes.

ceded by a period of glucose intolerance), obesity, insulin resistance and associated cardiovascular risk factors are its usual features. ${ }^{2}$ Type 1 diabetes is often thought to be a condition of lean adolescents and young adults that may be associated with other autoimmune diseases. More than $80 \%$ of patients with type 1 diabetes harbour markers of $\beta$ cell autoimmunity, including antibodies to glutamic acid decarboxylase and islet antigen-2. ${ }^{3}$ At diagnosis, C-peptide levels, indicative of endogenous insulin production, are nearly undetectable, whereas in most individuals with type 2 diabetes, endogenous insulin production is preserved, albeit decreasing, until late in the disease course.

Some individuals are not easily classified as having either type 1 or type 2 diabetes and may have overlapping features of both. Individuals with phenotypic type 2 diabetes do sometimes present with de novo ketoacidosis. Those with phenotypic type 1 diabetes who do well on oral agents or require minimal insulin may also present potential diagnostic confusion. Molecular diagnostic capabilities have confirmed a heterogeneous spectrum of diabetes. It is important to classify these forms accurately because the diagnosis of particular molecular subtypes carries important implications for predicting disease progression, considering the prognosis, making decisions about optimal treatment and possibly counselling the affected individual and family members regarding heritability of the disease $\mathrm{e}^{1,4}$

We review the approach to making an accurate diagnosis in patients with atypical or "intermediate" forms of diabetes that may masquerade as the common type 1 and type 2 diabetes phenotypes, with particular reference to monogenic diabetes (also referred to as maturity-onset diabetes of the young or MODY), ketosis-prone diabetes and latent autoimmune diabetes of adulthood. Box 1 outlines the evidence used in this review.

\section{General diagnostic considerations}

Accurately recognizing individuals with atypical diabetes is not always straightforward, nor is it an exact science. Often the diagnosis is suspected on the basis of unusual clinical features and only becomes clear once the natural history 
of the condition itself has become apparent. Clinicians caring for individuals with diabetes must first acknowledge that "other" forms of diabetes exist, so they can recognize the salient and sometimes subtle clinical features that define a particular phenotype. Although mutations in certain genes correlating with the clinical features of a monogenic diabetes phenotype can be considered diagnostic, in many instances the "atypical" diagnosis is suspected clinically through recognition of a characteristic phenotype that differs from typical type 1 or type 2 diabetes. Table $1^{3,5-8}$ compares the clinical features of type 1 diabetes, type 2 diabetes, hepatocyte nuclear factor $1 \alpha$ (HNF1A) diabetes, ketosis-prone diabetes and latent autoimmune diabetes of adulthood. Box 2 provides a case-based example of how clinical and laboratory features can be used to identify a patient with atypical diabetes.

\section{Monogenic diabetes}

Monogenic diabetes or MODY accounts for about $1 \%-2 \%$ of all cases of diabetes. ${ }^{9}$ Before the advent of molecular genetic diagnostic testing, this form of diabetes was believed to constitute a form of maturity-onset diabetes (i.e., type 2 diabetes) occurring at an unusually young age. Affected patients lack severe manifestations of absolute insulin deficiency, which distinguishes them from patients with the classic juvenile form of diabetes, now termed type 1 diabetes. ${ }^{10}$ This form of diabetes is often mislabelled as "lean" type 2 diabetes.

Monogenic diabetes is a heterogeneous group of disorders involving a variety of single-gene mutations in transcription factors or glycolytic

\section{Box 1: Evidence used in this review}

The goal of our review was to provide a practical clinical approach to a heterogeneous group of disorders, while paying particular attention to the more common forms of atypical diabetes that are likely to be encountered in general practice. Therefore, we were interested in articles about monogenic diabetes or maturity-onset diabetes of the young (MODY), in particular HNF1A diabetes (MODY 3), as well as ketosis-prone diabetes and latent autoimmune diabetes of adulthood. We searched MEDLINE (from 1948 to January 2013) and Google Scholar for relevant English-language articles using the following search terms: "atypical diabetes," "monogenic diabetes," "MODY," "HNF1A diabetes," "ketosis-prone diabetes," "latent autoimmune diabetes" and "LADA." We included both primary research articles and pertinent review articles. We also reviewed the reference lists of pertinent studies. Small case series and isolated case reports are common in this subject area, and we reviewed this literature, particularly for descriptions of novel therapeutic options. Given the emerging nature and heterogeneity of many atypical forms of diabetes, as well as the clinical overlap among them, the quality of the literature is highly variable. We included literature from major research groups that we believed to be of particular relevance to the topic. We placed particular emphasis on larger case series, cohort studies, clinical intervention trials and basic molecular pathophysiology literature, where available.

Table 1: Clinical comparison between type 2 diabetes mellitus, type 1 diabetes mellitus, HNF1A diabetes, ketosis-prone diabetes and latent autoimmune diabetes of adulthood ${ }^{3,5-8}$

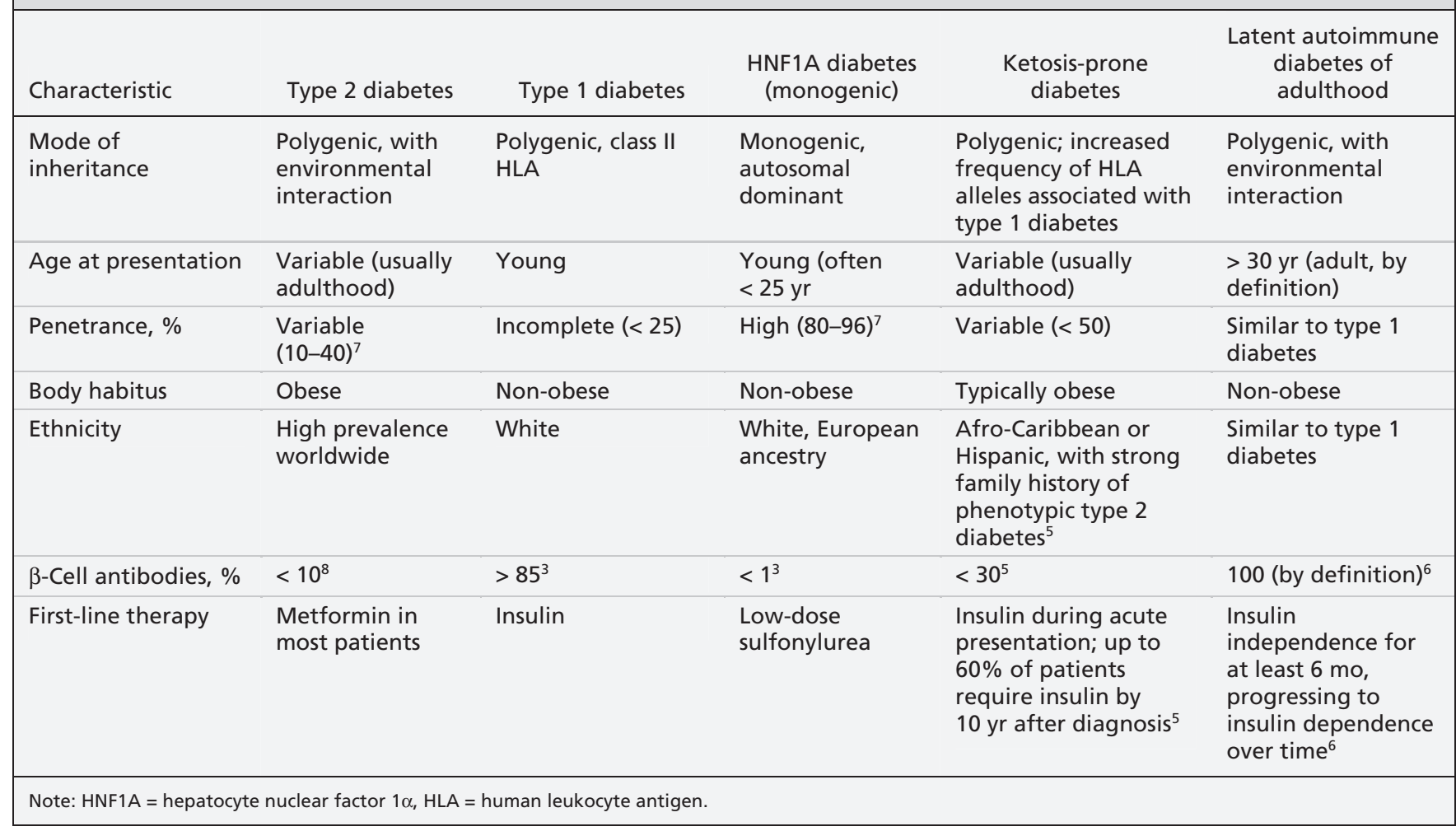


enzymes involved in $\beta$ cell glucose sensing and metabolism. To date, 11 subtypes have been described, and all are associated with a $\beta$ cell defect, although the subtypes differ in terms of clinical phenotype and spectrum of associated conditions. ${ }^{11}$ The most common forms relate to mutations in the genes encoding HNF1A (also termed MODY 3) and glucokinase (also termed MODY 2). These two subtypes account for about $80 \%$ of cases. HNF1A diabetes is the most common, occurring in up to $60 \%$ of individuals of European ancestry with monogenic diabetes. ${ }^{11}$ The estimated minimum prevalence for all diagnoses of MODY in a UK referral population was reportedly 108 cases per million. ${ }^{12}$ Regional variation in prevalence for all MODY subtypes is common. ${ }^{12}$ However, the prevalence is likely underestimated, with regional variation resulting from differences in referral rates, which in turn reflect extent of awareness, misdiagnosis or variation in access to genetic testing. ${ }^{12}$ Further discussion here is limited to HNF1A monogenic diabetes (MODY 3).

\section{Box 2: Applying the results of this review in clinical practice (fictional case)}

A 25-year-old man, at his initial visit with you, explains that he has had diabetes since he was 17 years old. His father and his only brother were also given a diagnosis of diabetes in their late teens. All were initially believed to have type 1 diabetes and were started on insulin as initial therapy at the time of diagnosis. The diagnosis seemed certain, given their young age, lean build and white ethnicity, with no signs of insulin resistance (which would have suggested type 2 diabetes). However, your patient's insulin dose has always been unusually low ( $<10$ units/d, with no basal insulin needed.) His fasting blood glucose is usually below $6 \mathrm{mmol} / \mathrm{L}$, and he is able to miss insulin doses occasionally, especially if he eats low-carbohydrate meals. He is prone to hypoglycemia when he increases his insulin or when he is exercising. No episodes of diabetic ketoacidosis have been reported.

On physical examination, he appears healthy, lean (body mass index 22) and normotensive; he has no acanthosis nigricans, nor evidence of diabetic retinopathy or neuropathy. His glycated hemoglobin (A1C) is $6.5 \%$ (normal for those without diabetes $<5.7 \%$ ), low-density lipoprotein cholesterol $2.1 \mathrm{mmol} / \mathrm{L}$ (target for people with diabetes $<2.6 \mathrm{mmol} / \mathrm{L}$ ) and high-density lipoprotein (HDL) cholesterol $1.3 \mathrm{mmol} / \mathrm{L}$ (target for men $>1 \mathrm{mmol} / \mathrm{L}$ ). His father has recently stopped all insulin and is now doing well on low-dose glimepiride therapy (1 mg/day). The patient asks, "Do I actually have type 2 diabetes?" He seeks your opinion regarding his diagnosis, prognosis and management.

This young, lean white man has all the features consistent with a diagnosis of hepatocyte nuclear factor $1 \alpha$ (HNF1A) monogenic diabetes: a strong multigenerational history of early-onset diabetes, minimal requirement for insulin, optimal fasting glycemic control, no episodes of diabetic ketoacidosis or signs of insulin resistance, and HDL cholesterol level inconsistent with the usual low levels seen in diabetic dyslipidemia. His father has responded very well to low-dose sulfonylurea therapy, in keeping with the heightened sensitivity to these agents that has been observed in HNF1A diabetes.

In summary, this patient should be told that he has an unusual form of diabetes that is strongly inherited. Genetic testing for the common HNF1A mutations is commercially available and could be offered to confirm the diagnosis. His risk of long-term complications is similar to that of similar patients with type 1 diabetes. He can be switched from insulin to a low-dose oral sulfonylurea drug and should be counselled as to the potential impact of his diagnosis on future family planning.
The hallmark of monogenic diabetes is autosomal dominant transmission, with high penetrance in families leading to multiple affected generations. Whereas individuals with type 2 diabetes usually have $30 \%-40 \%$ of first-degree relatives similarly affected, monogenic diabetes affects $50 \%$ of children born to a mutation carrier, and penetrance is often above $90 \%$, such that three or more generations are usually affected. ${ }^{11}$

HNF1A diabetes is characterized clinically by marked postprandial hyperglycemia, with fasting glucose tolerance relatively preserved early in the disease course. ${ }^{10}$ This feature makes early diagnosis solely on the basis of fasting glucose measurements challenging, especially in adolescence or childhood, when fasting glucose values are often normal. A suggestive diagnostic feature of HNF1A diabetes is the large incremental glucose response seen after a standard oral glucose load. More specifically, a glucose increment greater than $3 \mathrm{mmol} / \mathrm{L}$ at two hours after ingestion of $75 \mathrm{~g}$ oral glucose suggests HNF1A diabetes, especially if the fasting plasma glucose is less than $5.5 \mathrm{mmol} / \mathrm{L}$. This pattern contrasts with glucokinase diabetes, in which the two-hour increment is small $(<3 \mathrm{mmol} / \mathrm{L})$, fasting glucose is invariably above $5.5 \mathrm{mmol} / \mathrm{L}$, and diagnosis is more often based on the results of fasting glucose results rather than two-hour oral glucose tolerance test. ${ }^{13}$ Notably, the diagnosis would be missed in as many as $60 \%$ of individuals with HNF1A diabetes if glucose tolerance testing were omitted in favour of fasting glucose measurement alone. ${ }^{13}$ However, insulin secretion is progressively reduced at all glucose concentrations, and fasting hyperglycemia often develops over time. Therefore, certain suggestive features, as described in Table 2,,$^{3,13-18}$ should prompt formal genetic testing to establish the diagnosis. Increased insulin sensitivity may explain the lower-than-expected insulin requirements and proclivity to hypoglycemia with low-dose therapies. ${ }^{14}$

\section{Which diagnostic tests are useful?}

Potentially helpful laboratory tests in HNF1A diabetes include a lipid panel, ${ }^{17}$ oral glucose tolerance testing, ${ }^{13}$ urinalysis for glucose ${ }^{14}$ and highsensitivity testing for $\mathrm{C}$-reactive protein, ${ }^{18}$ although none of these is considered diagnostic. Islet cell antibodies (i.e., antibodies to glutamic acid decarboxylase and/or islet antigen-2) are usually absent in HNF1A diabetes (positive result in $<1 \%$ of individuals), which provides reliable discrimination from type 1 diabetes (positive result in $>85 \%$ ) and latent autoimmune diabetes of adulthood. Therefore, antibody testing should be routinely performed before more 
expensive genetic testing. ${ }^{3}$ Where available, genetic testing should be used to confirm a specific diagnosis of monogenic diabetes.

\section{What are the benefits and implications of diagnosis?}

HNF1A diabetes carries a risk for complications of poor glycemic control similar to that of type 1 diabetes. ${ }^{19}$ Despite frequent absence of associated cardiovascular risk factors, cardiovascular morbidity is higher and life expectancy lower than among matched nonaffected family members. ${ }^{16}$ However, the $\beta$ cell defect is often managed successfully for years with insulin secretagogue therapies, such as sulfonylurea or meglitinide drugs. Because of increased insulin sensitivity, individuals may have exquisite sensitivity to the insulin secretagogue drugs. The lowest available dose of a particular sulfonylurea drug is often adequate to treat HNF1A diabetes, especially early in the disease course; however, certain individuals may continue to struggle with hypoglycemia despite careful dose titration. ${ }^{15,20}$

The role of incretin mimetic drugs, such as glucagon-like peptide-1 agonists or the weaker dipeptidyl peptidase-4 inhibitors, has yet to be established, and very few prospective data are available to inform their use. Nateglinide, a short-acting meglitinide-class secretagogue, has been prospectively studied in a small group of patients with HNF1A diabetes; it was associated with effective postprandial glucose control, lower peak insulin concentrations and less hypoglycemia than low-dose sulfonylurea therapy. ${ }^{21-23}$ Metformin and thiazolidinediones are much less effective and do not address the underlying pathophysiologic defect of impaired insulin secretion; as such, they are rarely indicated..$^{20}$

Insulin may be required to achieve glycemic control during periods of reduced insulin sensitivity (e.g., pregnancy), in acute illness or when oral secretagogue agents are no longer effective because of the often progressive natural history of HNF1A diabetes. Prandial rapid-acting insulin at small doses may be all that is needed, given the heightened insulin sensitivity and often well-maintained fasting glucose values. In addition, the $50 \%$ chance of having an affected child ${ }^{7}$ carries implications for family planning and genetic counselling. Table 2 outlines clinical considerations and laboratory findings in HNF1A diabetes. ${ }^{3,13-18}$

\section{Ketosis-prone diabetes}

Ketosis-prone diabetes is seen in an emerging, heterogeneous group of individuals in whom diabetic ketoacidosis develops without the typical phenotype of type 1 diabetes. However, differentiation of ketosis-prone diabetes from type 1 diabetes in older, obese, nonwhite individuals can be challenging, and the correct diagnosis may be

Table 2: Clinical and laboratory features of HNF1A diabetes (maturity-onset diabetes of the young type 3$)^{3,13-18}$

\begin{tabular}{|c|c|}
\hline Clinical considerations & Laboratory features \\
\hline $\begin{array}{l}\text { Autosomal dominant inheritance; often more } \\
\text { than three generations with diabetes }\end{array}$ & C- peptide detectable \\
\hline High penetrance in families & Islet cell antibodies usually absent ${ }^{3}$ \\
\hline European ethnicity most common & Normal triglyceride levels \\
\hline Lean (BMI often < 25) & Normal or high HDL cholesterol levels ${ }^{17}$ \\
\hline Usually young (<25 yr) & $\begin{array}{l}\text { Low C-reactive protein levels with high-sensitivity } \\
\text { testing }{ }^{18}\end{array}$ \\
\hline No ketoacidosis & $\begin{array}{l}\text { Low renal threshold for glucose; glucosuria often } \\
\text { occurs when serum glucose }>8 \mathrm{mmol} / \mathrm{L}^{14}\end{array}$ \\
\hline $\begin{array}{l}\text { Increased insulin sensitivity; patients often have } \\
\text { minimal requirement for insulin }{ }^{14}\end{array}$ & $\begin{array}{l}\text { Large ( }>3.0 \mathrm{mmol} / \mathrm{L}) \text { rise in } 2 \text {-h glucose levels on } \\
75-\mathrm{g} \text { oral glucose tolerance testing }\end{array}$ \\
\hline Hypersensitivity to sulfonylurea drugs ${ }^{15}$ & $\begin{array}{l}\text { For up to } 50 \% \text { of patients, fasting glucose } \\
<5.5 \mathrm{mmol} / \mathrm{L} \text { early in disease course } \mathrm{e}^{13}\end{array}$ \\
\hline \multicolumn{2}{|l|}{ Postprandial hyperglycemia dominates } \\
\hline \multicolumn{2}{|l|}{$\begin{array}{l}\text { Prevalence of microvascular and macrovascular } \\
\text { complications similar to that of patients with } \\
\text { type } 1 \text { diabetes }^{16}\end{array}$} \\
\hline $\begin{array}{l}\text { Progressive } \beta \text {-cell failure over time, with increased } \\
\text { fasting glucose } \mathrm{e}^{13}\end{array}$ & \\
\hline
\end{tabular}


discerned only with careful follow-up. Ketoacidosis follows severe $\beta$ cell dysfunction with marked insulin deficiency. Affected patients often present with an episode of unprovoked diabetic ketoacidosis and are discharged from hospital on insulin therapy. However, in certain individuals, this acute dysfunction is transient and is followed by robust $\beta$ cell recovery, with complete resolution of exogenous insulin requirement and near normoglycemia. Over periods of months to years, these individuals may "swing" between periods of acute insulin deficiency and subsequent "remission" characterized by milder dysglycemia, with minimal to no need for antihyperglycemic agents. ${ }^{24}$

Ketosis-prone diabetes has been variably termed "atypical," "Flatbush," "reversible" or "ketosis-prone type 2 diabetes," which reflects the ongoing difficulty of classifying this heterogeneous group..$^{24} \mathrm{~A}$ useful classification system, designated the $A B$ classification scheme, has been proposed. In this system, individuals are categorized into one of four groups, depending on the presence or absence of islet cell autoantibodies (A+ or A-, respectively) and the presence or absence of $\beta$ cell functional reserve once the period of acute metabolic decompensation has resolved ( $\mathrm{B}+$ or $\mathrm{B}-$, respectively). ${ }^{24} \mathrm{~A}+\mathrm{B}-$ and $\mathrm{A}-\mathrm{B}-$ individuals are distinct subgroups with differing genetic and immunologic underpinnings, but they share the clinical characteristics of type 1 diabetes, including reduced $\beta$ cell secretory function. In contrast, $\mathrm{A}+\mathrm{B}+$ and $\mathrm{A}-\mathrm{B}+$ individuals share the features of type 2 diabetes, with preservation of $\beta$ cell function over time. ${ }^{25}$

Patients in the largest of these four groups
$(\mathrm{A}-\mathrm{B}+)$ resemble people with the type 2 diabetes obese phenotype and account for $50 \%$ of all those with ketosis-prone diabetes. ${ }^{25}$ Unprovoked diabetic ketoacidosis, with absent islet cell antibodies and frequent evolution to insulin independence, is the hallmark of this group. About half of patients will become insulin independent after an early episode of diabetic ketoacidosis, but ultimately $60 \%$ will be insulin dependent 10 years after diagnosis. ${ }^{26}$ Individuals are often of Afro-Caribbean or Hispanic ancestry, but ketosis-prone diabetes has been described in many ethnic groups worldwide. Male predominance is another hallmark of the group with unprovoked diabetic ketoacidosis. ${ }^{5}$ The exact prevalence of ketosis-prone diabetes is unknown, given limited detailed epidemiologic data. However, this form of diabetes is being increasingly reported and recognized around the world. ${ }^{5}$

\section{Which diagnostic tests are useful?}

There are no gold standard diagnostic tests defining ketosis-prone diabetes. Islet cell antibodies and C-peptide levels should be obtained for all patients with suspected atypical phenotypes to evaluate for markers of $\beta$ cell autoimmunity and $\beta$ cell secretory reserve. ${ }^{25,27}$ Referral to an endocrinology specialist is often appropriate to aid in establishing the specific cause and for help in formulating an approach to treatment.

\section{What are the benefits and implications of diagnosis?}

The main clinical relevance of diagnosing ketosis-prone diabetes is in distinguishing individuals with this form of diabetes from those with type 1 diabetes, who will require lifelong insulin

Table 3: Clinical and laboratory features of ketosis-prone diabetes $5,25,27$

\begin{tabular}{|ll|}
\hline Clinical considerations & \multicolumn{1}{c|}{ Laboratory features } \\
\hline $\begin{array}{l}\text { Unprovoked ketoacidosis often occurs; may be } \\
\text { new-onset diabetes }\end{array}$ & $\beta$-cell antibodies present in up to $28 \%$ of patients ${ }^{25}$ \\
\hline Afro-Caribbean or Hispanic ancestry & $\begin{array}{l}\text { C-peptide often low or undetectable during } \\
\text { diabetic ketoacidosis; recovery expected in }>60 \%\end{array}$ \\
\hline $\begin{array}{l}\text { Periods of insulin independence interspersed } \\
\text { with periods of acute insulin deficiency and } \\
\text { diabetic ketoacidosis }\end{array}$ & $\begin{array}{l}\text { Ratio of fasting C-peptide (nmol/L) to glucose } \\
\text { (mmol/L) }>11 \text { may be used as reliable predictor of } \\
\text { insulin discontinuation }{ }^{27}\end{array}$ \\
\hline $\begin{array}{l}\text { Type } 2 \text { diabetes phenotype common (obesity, } \\
\text { insulin resistance, metabolic syndrome) }\end{array}$ & $\begin{array}{l}\text { Presence of HLA alleles for type } 1 \text { diabetes } \\
\text { associated with insulin dependence within } 1-2 \text { yr }^{25}\end{array}$ \\
$\begin{array}{l}\text { Fluctuating glycated hemoglobin (A1C) pattern } \\
\text { consistent with } \beta \text {-cell failure and recovery }\end{array}$ & \\
\hline $\begin{array}{l}\text { Male predominance among patients with } \\
\text { unprovoked diabetic ketoacidosis (male-female } \\
\text { ratio } 2.6: 1)^{5}\end{array}$ & \\
\hline $\begin{array}{l}\text { Insulin should always be initial therapy, with } \\
\text { reduction of cardiovascular risk factors }\end{array}$ & \\
\hline Note: HLA = human leukocyte antigen.
\end{tabular}


therapy. $\beta$ cell recovery is not easy to predict, given the heterogeneity of this group. However, for individuals with new-onset, unprovoked diabetic ketoacidosis and absent islet cell autoantibodies, the rate of insulin discontinuation is favourable. Of the three specific factors that may predict $\beta$ cell recovery and insulin discontinuation in this subgroup - new-onset diabetes, onset of diabetes in middle age and significant $\beta$ cell functional reserve (ratio of C-peptide $[\mathrm{nmol} / \mathrm{L}]$ to glucose $[\mathrm{mmol} / \mathrm{L}]>11$ ) - the third is the strongest predictor. ${ }^{27}$ If insulin is successfully withdrawn, insulin sensitizers such as metformin and thiazolidinediones are often appropriate first-line agents. ${ }^{5}$ Table $3^{5,25,27}$ outlines clinical considerations and laboratory findings in ketosis-prone diabetes.

\section{Latent autoimmune diabetes of adulthood}

Latent autoimmune diabetes of adulthood is perhaps the least well defined and most confusing form of atypical diabetes. Affected patients generally include adults with phenotypic type 2 diabetes and detectable islet cell antibodies, who present without ketoacidosis or catabolism. In simplified terms, this condition is slowly progressive autoimmune type 1 diabetes that is usually diagnosed at an older age than typical type 1 diabetes. To add further confusion, latent autoimmune diabetes of adulthood has also been called "slowly progressive type 1 diabetes," "latent type 1 diabetes," "double diabetes" and "type 1.5 diabetes." Additionally, there may be significant clinical overlap with ketosis-prone diabetes, which again highlights the imperfect clinical discrimination of current diabetes classification. The American Diabetes Association does not consider this form of diabetes distinct from type 1 diabetes. ${ }^{1}$ A somewhat arbitrary and imprecise definition proposed by the Immunology of Diabetes Society (in an effort to facilitate study) lists the following criteria: onset of diabetes at older than 30 years of age, presence of at least one of the four islet cell antibodies common to type 1 diabetes and insulin independence for at least six months after diagnosis. ${ }^{6}$

These individuals are typically older and may even present with antibody-positive diabetes in the seventh or eighth decade of life. In fact, recent studies have suggested that autoimmune diabetes presenting in adulthood most commonly presents as latent autoimmune diabetes of adulthood. ${ }^{28}$ Hyperglycemia is initially controlled with oral antihyperglycemic therapy, but over months to years patients progress to insulin dependence. ${ }^{29}$ Latent autoimmune diabetes of adulthood may well be a truly "intermediate" form of diabetes, as certain genetic features common to both type 1 and type 2 diabetes are present. ${ }^{30}$ However, the clinical utility of diagnosing this form of diabetes is highly debated..$^{31,32}$

\section{Which diagnostic tests are useful?}

As with ketosis-prone diabetes, there are no gold standard diagnostic tests that define latent autoimmune diabetes of adulthood. However, positive markers of $\beta$ cell autoimmunity are required, and measurement of $\beta$ cell secretory reserve (i.e., C-peptide) is useful to predict insulin secretory capacity.

\section{What are the benefits and implications of diagnosis?}

The controversial clinical entity of latent autoimmune diabetes of adulthood constitutes the most common presentation of autoimmune diabetes in adults. The value of considering this diagnosis lies in recognizing its prevalence in the growing epidemic of type 2 diabetes. In particular, $\beta$ cell autoimmunity may be found in phenotypic type 2 diabetes. Even if only $10 \%$ of cases of phenotypic type 2 diabetes are latent autoimmune diabetes of adulthood, this represents a much larger autoimmune diabetes burden than is represented by confirmed childhood type 1 diabetes. These individuals progress to insulin dependence faster than patients with antibody-negative type 2 diabetes.

Table 4: Clinical and laboratory features of latent autoimmune diabetes of adulthood ${ }^{6,30,33}$

Clinical considerations

Laboratory features

Age $>30 \mathrm{yr}$ GAD and ICA antibodies present

Patients may be overweight, but typically leaner than those with type 2 diabetes

Patients may have mild to moderate insulin resistance ${ }^{27}$ Insulin antibodies often absent

Autoimmune markers may show subtle differences from those of childhood type 1 diabetes, but are more similar to antibodies seen in adult-onset type 1 diabetes $^{29}$

Occurs in people of various ethnicities

Progression to insulin therapy slower

than for patients with type 1

diabetes, but quicker than for those

with type 2 diabetes

Avoid $\beta$-cell stressors such as secretagogues $^{32}$

May be treated initially with oral

antihyperglycemic agents, but insulin

should be introduced early if glycemic

control cannot be maintained

Note: GAD = glutamic acid decarboxylase, ICA = islet cell antibodies 
Thus, insulin treatment may be introduced earlier in the disease course, for the theoretical benefit of correcting glucotoxicity and possibly prolonging $\beta$ cell reserve. Drugs with potential deleterious effects on $\beta$ cell function, such as sulfonylureas, are best avoided..$^{33}$ Table $4^{6,27,29,30,32,33}$ outlines clinical considerations and laboratory findings in latent autoimmune diabetes of adulthood.

\section{Conclusion}

Although the various forms of atypical diabetes are relatively uncommon, it is important that they be accurately diagnosed, as diagnosis may have a substantial impact on the prognosis and management of individual patients and their families. However, for both clinicians and patients, it is often less important to label the specific type of diabetes than it is to understand the pathophysiology of the hyperglycemia to allow effective treatment.

\section{References}

1. American Diabetes Association. Standards of medical care in diabetes 2012. Diabetes Care 2012;35(Suppl 1):S11-63.

2. DeFronzo RA, Bonadonna RC, Ferrannini E. Pathogenesis of NIDDM. A balanced overview. Diabetes Care 1992:15:318-68.

3. McDonald TJ, Colclough K, Brown R, et al. Islet autoantibodies can discriminate maturity-onset diabetes of the young (MODY) from type 1 diabetes. Diabet Med 2011;28:1028-33.

4. Thethy I, Robertson L, Swaminathan K. Diagnosis in diabetes: Does it matter? J R Coll Physicians Edinb 2011;41:119-21.

5. Balasubramanyam A, Nalini R, Hampe CS, et al. Syndromes of ketosis-prone diabetes mellitus. Endocr Rev 2008;29:292-302.

6. Naik RG, Brooks-Worrell BM, Palmer JP. Latent autoimmune diabetes in adults. J Clin Endocrinol Metab 2009;94:4635-44.

7. Fajans SS, Bell GI, Polonsky KS. Molecular mechanisms and clinical pathophysiology of maturity-onset diabetes of the young. N Engl J Med 2001;345:971-80.

8. Wroblewski M, Gottsäter A, Lindgärde F, et al. Gender, autoantibodies, and obesity in newly diagnosed diabetic patients aged 40-75 years. Diabetes Care 1998;21:250-5.

9. Murphy R, Ellard S, Hattersley AT. Clinical implications of a molecular genetic classification of monogenic B-cell diabetes. Nat Clin Pract Endocrinol Metab 2008;4:200-13.

10. Tattersall RB, Fajans SS. A difference between the inheritance of classical juvenile-onset and maturity-onset type diabetes of young people. Diabetes 1975;24:44-53.

11. Fajans SS, Bell GI. MODY: history, genetics, pathophysiology, and clinical decision making. Diabetes Care 2011;34:1878-84.

12. Shields BM, Hicks S, Shepherd MH, et al. Maturity-onset diabetes of the young (MODY): How many cases are we missing? Diabetologia 2010;53:2504-8.

13. Stride A, Vaxillaire M, Tuomi T, et al. The genetic abnormality in the beta cell determines the response to an oral glucose load. Diabetologia 2002;45:427-35.

14. Stride A, Ellard S, Clark P, et al. Beta-cell dysfunction, insulin sensitivity, and glycosuria precede diabetes in hepatocyte nuclear factor-1alpha mutation carriers. Diabetes Care 2005;28: 1751-6.

15. Søvik O, Njolstad P, Folling I, et al. Hyperexcitability to sulfonylurea in MODY3. Diabetologia 1998;41:607-8.

16. Steele AM, Shields BM, Shepherd M, et al. Increased all-cause and cardiovascular mortality in monogenic diabetes as a result of mutations in the HNF1A gene. Diabet Med 2010;27:157-61.

17. Pearson ER. HDL-cholesterol: differentiating between HNF1A MODY and type 2 diabetes [abstract]. Diabet Med 2003;20 (Suppl 22):S21-33.

18. McDonald TJ, Shields BM, Lawry J, et al. High-sensitivity CRP discriminates HNF1A-MODY from other subtypes of diabetes. Diabetes Care 2011;34:1860-2.

19. Isomaa B, Henricsson M, Lehto M, et al. Chronic diabetic complications in patients with MODY3 diabetes. Diabetologia 1998:41:467-73

20. Pearson ER, Starkey BJ, Powell RJ, et al. Genetic cause of hyperglycaemia and response to treatment in diabetes. Lancet 2003;362:1275-81.

21. Shepherd M, Pearson ER, Houghton J, et al. No deterioration in glycemic control in HNF-1alpha maturity-onset diabetes of the young following transfer from long-term insulin to sulphonylureas. Diabetes Care 2003;26:3191-2.

22. Shepherd M, Shields B, Ellard S, et al. A genetic diagnosis of HNF1A diabetes alters treatment and improves glycaemic control in the majority of insulin-treated patients. Diabet Med 2009; 26:437-41.

23. Tuomi T, Honkanen EH, Isomaa B, et al. Improved prandial glucose control with lower risk of hypoglycemia with nateglinide than with glibenclamide in patients with maturity-onset diabetes of the young type 3. Diabetes Care 2006;29:189-94.

24. Balasubramanyam A, Garza G, Rodriguez L. at al. Accuracy and predictive value of classification schemes for ketosis-prone diabetes. Diabetes Care 2006;29:2575-9.

25. Maldonado M, Hampe CS, Gaur LK, et al. Ketosis-prone diabetes: dissection of a heterogeneous syndrome using an immunogenetic and B-cell functional classification, prospective analysis, and clinical outcomes. J Clin Endocrinol Metab 2003;88:5090-8.

26. Mauvais-Jarvis F, Sobngwi E, Porcher R, et al. Ketosis-prone type 2 diabetes in patients of sub-Saharan African origin: clinical pathophysiology and natural history of B-cell dysfunction and insulin resistance. Diabetes 2004;53:645-53.

27. Maldonado MR, Otiniano ME, Cheema F, et al. Factors associated with insulin discontinuation in subjects with ketosis-prone diabetes but preserved B-cell function. Diabet Med 2005;22: 1744-50.

28. Hawa MI, Kolb H, Schloot N, et al. Adult-onset autoimmune diabetes in Europe is prevalent with a broad clinical phenotype: action LADA 7. Diabetes Care 2013;36:908-13.

29. Kobayashi T, Tamemoto K, Nakanishi K, et al. Immunogenetic and clinical characterization of slowly progressive IDDM. Diabetes Care 1993;16:780-8

30. Cervin C, Lyssenko V, Bakhtadze E, et al. Genetic similarities between latent autoimmune diabetes in adults, type 1 diabetes, and type 2 diabetes. Diabetes 2008;57:1433-7.

31. Palmer JP, Hirsch IB. What's in a name: latent autoimmune diabetes of adults, type 1.5, adult-onset, and type 1 diabetes. Diabetes Care 2003;26:536-8.

32. Palmer JP, Hampe CS, Chiu H, et al. Is latent autoimmune diabetes in adults distinct from type 1 diabetes or just type 1 diabetes at an older age? Diabetes 2005;54(Suppl 2):S62-7.

33. Kobayashi T, Maruyama T, Shimada A, et al. Insulin intervention to preserve B cells in slowly progressive insulin dependent (type 1) diabetes mellitus. Ann N Y Acad Sci 2002;958:117-30.

Affiliation: Section of Endocrinology, Diabetes and Nutrition, Boston Medical Center, and Boston University School of Medicine, Boston, Mass.

Contributors: Devin Steenkamp and Sara Alexanian conceived the article. Devin Steenkamp performed the initial literature search and reviewed and extracted relevant information from the included articles. Elliot Sternthal searched for and reviewed further relevant articles pertaining to latent autoimmune diabetes of adulthood. Devin Steenkamp drafted the article, which was revised by all authors. All of the authors approved the final version submitted for publication. 